\title{
Imaging for suspected pulmonary embolism in pregnancy-what about the fetal dose? A comprehensive review of the literature
}

\author{
Tilo Niemann • Guillaume Nicolas • Hans W. Roser • \\ Jan Müller-Brand • Georg Bongartz
}

Received: 12 April 2010 /Revised: 13 August 2010/Accepted: 15 September 2010 / Published online: 2 October 2010

(C) European Society of Radiology 2010

\begin{abstract}
Objective To give a comprehensive overview of fetal doses reported in the literature when imaging the pregnant woman with suspected pulmonary embolism (PE).

Methods A comprehensive literature search in the PubMed, MEDLINE and EMBASE databases yielded a total of 1,687 papers that were included in the analysis and have been analysed with regard to fetal dose in suspected PE radiological imaging strategies.

Results Fetal dose in chest computed tomography (CT) ranges between 0.013 and 0.026 mGy in early and 0.06$0.1 \mathrm{mGy}$ in late pregnancy compared with $99 \mathrm{mTc}-\mathrm{MAA}$ perfusion scintigraphy with a fetal dose of $0.1-0.6 \mathrm{mGy}$ in early and $0.6-0.8 \mathrm{mGy}$ in late pregnancy. ${ }^{99 \mathrm{~m}} \mathrm{Tc}$-aerosol ventilation scintigraphy results in $0.1-0.3 \mathrm{mGy}$. However, there is concern about female breast irradiation in CT, which is higher than in scintigraphy. CT radiation risks for breast tissue remain unclear.

Conclusion Knowledge of dosimetry and radiation risks is crucial in the radiological work-up of suspected PE in
\end{abstract}

\footnotetext{
T. Niemann $(\bowtie) \cdot G$. Bongartz

Department of Radiology, University Hospital,

Petersgraben 4 ,

4031 Basel, Switzerland

e-mail: niemannt@uhbs.ch

G. Nicolas $\cdot$ J. Müller-Brand

Department of Nuclear Medicine, University Hospital,

Petersgraben 4,

4031 Basel, Switzerland

H. W. Roser

Department of Radiology, Radiological Physics,

University Hospital,

Petersgraben 4,

4031 Basel, Switzerland
}

pregnancy. It is reasonable to reserve scintigraphy for pregnant patients with normal chest radiography findings and no history of asthma or chronic lung disease. Performing CT applying dose reduction instead of scintigraphy will minimise fetal radiation dose and maximise the diagnostic value.

Keywords Pulmonary embolism - Radiation protection . Tomography $\cdot$ Spiral computed $\cdot$ Radionuclide imaging

\section{Introduction}

Work-up of pulmonary embolism (PE) in pregnancy remains a diagnostic dilemma. The morbidity and mortality in the context of pregnancy-related PE in the developed world is well known [1-4]. In pregnancy the clinical diagnosis of both deep-vein thrombosis and PE is generally difficult and is further complicated because dyspnoea, tachypnoea, swelling and discomfort in the legs are common/expected symptoms of pregnancy and must be interpreted with caution. As the rate of PE in pregnancy is five-times higher than for non-pregnant women of the same age, precise PE diagnosis in pregnancy is vital [5].

In thecriteria of Wells et al. [6], the most frequently used tool for assessing the clinical probability of PE, pregnant patients were excluded in the validation of the criteria. Therefore estimating the pre-test probability for PE can be difficult. Even the D-dimers are known to show special dynamics during pregnancy [5]. New reference ranges for normal D-dimer levels for each trimester and novel serum markers such as the fibrin monomer complex may play a future role in the management of suspected PE in pregnant patients $[7,8]$. Therefore, estimating the pre-test probability for PE can be difficult. 
Unnecessary treatment of PE must be prevented as treatment is associated with probable and critical side effects for both mother and fetus [9].

In the literature there is controversy as to which radiological imaging strategy is optimal in suspected pregnancy-related PE with regard to fetal radiation dose.

The increasing use of CT causes concern about the safety and efficacy of this modern technology. The Safety and Efficacy in Computed Tomography (SECT) project, supported by the EC-EURATOM 6th Framework Program [10], is aimed at dose reduction by the justification and optimisation of CT. Recent publications support the concern expressed in the SECT project $[11,12]$.

In general, but especially when young or pregnant patients are concerned, it must be aimed at optimising policies in diagnostic imaging with regard to decreasing radiation dose as far as possible. In the assessment of suspected pulmonary embolism in pregnant patients, workup should begin with tests that provide diagnostic information with the use of little or no ionising radiation as chest radiography and ultrasound. If these tests prove to be nondiagnostic, further imaging such as lung scintigraphy or CT pulmonary angiography (CTA) should be performed $[9,13]$.

Currently there are two major roentgenological pathways in the radiological work-up of suspected PE in pregnancy. Before their application, clinical risk stratification and laboratory tests as well as lower limb compression ultrasound should be performed in all pregnant patients.

One main diagnostic algorithm is the scintigraphic workup with either combined ventilation/perfusion scans (V/Q) or solitary perfusion imaging. The other major pathway is the routinely performed CTA using dose reduction measures to protect the mother and especially the fetus from unnecessary radiation.

Recent surveys show that there is limited understanding and level of awareness among healthcare professionals about dosimetry and radiation risks associated with imaging pregnant women [14].

Understanding of the estimated exposure dose to the fetus permits rational and adequate choices of the imaging strategies. The purpose of this paper is to give an overview of the literature and compare the two major imaging strategies with regard to the fetal dose in suspected PE.

\section{Data extraction}

A comprehensive computer literature search of abstracts was performed to identify articles on fetal dosage in chest CT or scintigraphy for suspected PE. Initially the PubMed databases were used with the following search query:

("pulmonary embolism"[MeSH Terms] OR (("pulmonary"[tiab] OR "lung"[tiab]) AND “embolism"[tiab]))
AND ("pregnancy"[MeSH Terms] OR "pregnant"[tiab] OR "fetus"[MeSH Terms] OR "fetus"[tiab])

The initial search query yielded a total of 1,687 articles. The search query was translated accordingly for the MEDLINE (and EMBASE) databases, but retrieved no further articles.

The analysis was limited to papers published after 1990 with the rationale that before 1990 the technical equipment was substantially different to that currently used. The search was further restricted to the English, French, Spanish and German languages. Case reports were excluded. Two observers independently checked all retrieved studies for inclusion. Disagreements were resolved in consensus.

These limitations resulted in a total of 497 articles. Further title and abstract clearing of non-relevant articles yielded a total of 85 papers that could be used for the literature review (Fig. 1). Extensive cross-checking of references was performed.

\section{CTA pathway}

CTA has gained widespread acceptance as the new standard of reference for suspected PE in the general population because of its high sensitivity and specificity [15-20]. See Table 1 for an overview of the studies discussed.

Hurwitz et al. [21] used an anthropomorphic phantom to determine fetal doses from a range of multidetector-row CT

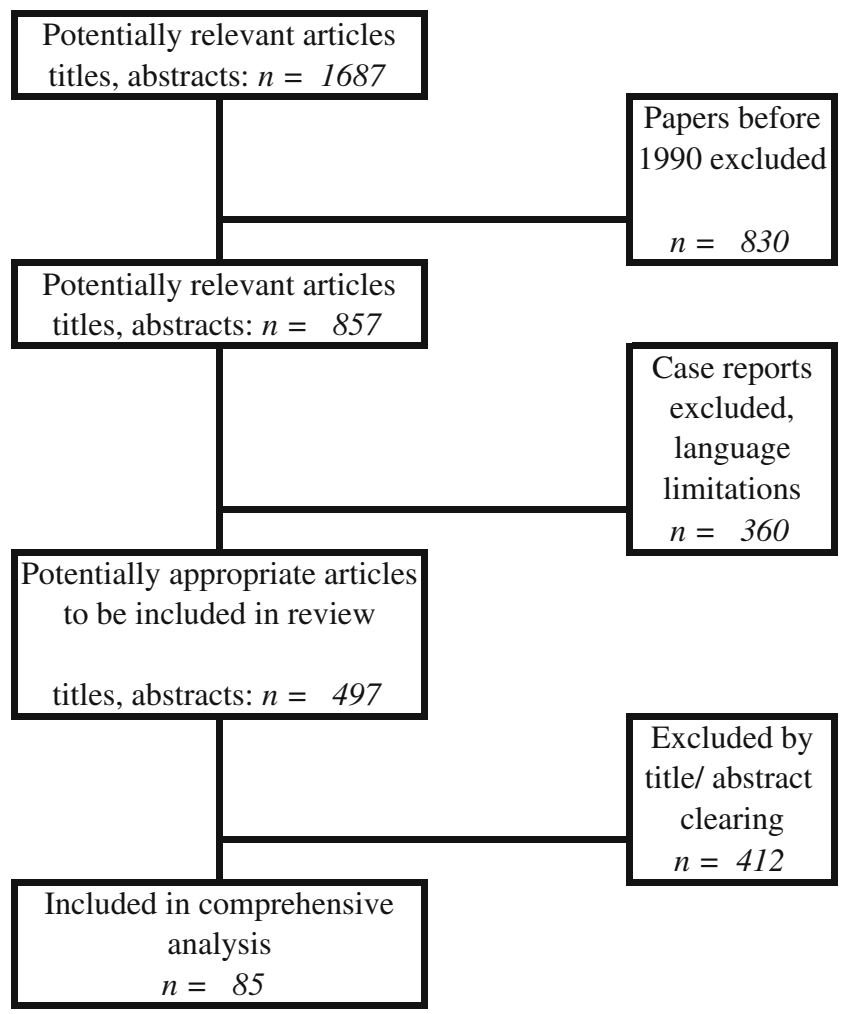

Fig. 1 Results of the comprehensive literature search 
investigations in the early stages of pregnancy. They varied the position of the uterus during the first three months of pregnancy. A routine CT protocol was applied for the simulations without any dose reduction measures $(140 \mathrm{kV}$, 380 mAs, DLP 507.3 mGy cm, CTDI ${ }_{\mathrm{vol}} 28.39$ mGy, 16× $1.25 \mathrm{~mm}$ collimation). The fetal radiation dose recorded from this protocol was less than $0.7 \mathrm{mGy}(0.24-0.47 \mathrm{mGy}$ for 0 months and $0.6-0.66 \mathrm{mGy}$ for 3 months of pregnancy).

Doshi et al. [22] used an anthropomorphic phantom to evaluate fetal dose in late pregnancy. The phantom underwent imaging with different equipment with varied technical parameters. Mean fetal absorbed dose was calculated as $0.23-0.6 \mathrm{mGy}$, whereas an imaging protocol using contemporary equipment and recent technical parameters resulted in a range of 0.06-0.1 mGy. Further fetal dose reduction could be accomplished using different dose reduction strategies. Automatic tube current modulation resulted in a 10\% further decrease in dose; anterior abdominal lead shielding (positioning the lead out of the imaging field) yielded a $35 \%$ dose reduction. Imaging with a $5-\mathrm{cm}$ shorter image length in the lung bases resulted in $55 \%$ dose reduction.

Another approach based on Monte Carlo simulations was performed by Winer-Muram et al. [23]. Maternal-fetal geometries of 23 patients were used for the analysis. A serial routine $\mathrm{CT}$ protocol was used for calculation of the imaging parameters $(120 \mathrm{kV}, 100 \mathrm{mAs})$. Mean fetal doses were estimated for all trimesters as follows: 0.00330.02 mGy first trimester; 0.0079-0.076 mGy second trimester; and 0.051-0.130 mGy, third trimester.

Nijkeuter et al. [24] applied published conversion factors to estimate the dose to the uterus and hence the fetus in early pregnancy. A standard chest CT protocol for 16-row multidetector (MD) CT was used for image acquisition $(120 \mathrm{kV}, 85 \mathrm{mAs})$. The equivalent dose to the fetus was in the region of $0.013 \mathrm{mGy}$. Using a single-row system $(120 \mathrm{kV}, 250 \mathrm{mAs})$ resulted in a fetal equivalent dose of $0.026 \mathrm{mGy}$. The calculations based on the assumption that the amount of radiation absorbed by the fetus was assumed to be equal to that absorbed by the uterus of a non-pregnant woman.

Another calculation performed by Huda [26] retrieved a fetal dose of $\sim 0.14 \mathrm{mGy}$. The trimester of pregnancy was not further specified, but, taking into account the location of the fetus of at least $20 \mathrm{~cm}$, later pregnancy must be assumed. The data were based on the dose measurements of Boone et al. [25]. A tube voltage of $120 \mathrm{kV}$ was used and $150 \mathrm{mAs}$ tube current, the system type was not further specified.

Damilakis et al. [27] provided fetal dose measurements using anthropomorphic phantoms for third trimester simulations. For chest $\mathrm{CT}$ examination they used a standard protocol $(120 \mathrm{kV}, 200 \mathrm{mAs})$ for a single slice system. Dose 
calculations based on manual segmentation of the fetal position resulted in a mean fetal dose of $1.2 \mathrm{mGy}$ for late pregnancy.

Cook and Kyriou [28] reported an absorbed dose to the fetus of $0.01 \mathrm{mGy}$ for CTA without specifying the trimester. No technical parameters for the CT protocol used or any scanner details were specified.

The International Commission of Radiation Protection (ICRP) further published mean fetal doses for different radiological examinations. The approximate mean fetal dose from chest CT was reported to be $0.06 \mathrm{mGy}$ [29] for single-detector-row helical CT. No further technical specifications were mentioned, nor the trimester specified.

Mean fetal doses/estimates of uterine dose published by the National Radiological Protection Board (NRPB) are noted as $0.06 \mathrm{mGy}$ using a single-slice system [30] without further specifying imaging parameters or the trimester.

\section{Scintigraphy pathway}

In the era of CTA ventilation-perfusion (V/Q) imaging has become the second-line imaging technique for suspected PE. As many other conditions such as pneumonia or bronchospasms may cause perfusion defects in lung scintigraphy ventilation imaging is used routinely to match/mismatch these defects [31].

In many centres, the relatively low incidence of lung comorbidity in pregnancy allows perfusion scintigraphy to be performed using "half-dose" (40 $\left.\mathrm{MBq}{ }^{99 \mathrm{~m}} \mathrm{Tc}-\mathrm{MAA}\right)$ without the need for ventilation imaging [29, 32]; preselection of these patients based on normal chest radiography is important to prevent non-diagnostic images. Extending imaging duration may enhance image quality in this context of dose-reduced lung perfusion. The interpretation of lung perfusion scintigraphy is usually straightforward in pregnant patients because of the low frequency of comorbid pulmonary disorders [33].

In nuclear medicine, fetal doses depend predominantly upon the administered activity. In very ill patients there may be individual differences in metabolism and distribution of the radiopharmaceuticals. Conversely, pregnant women in most cases have essentially normal distribution of radiopharmaceuticals and fetal doses can be reasonably estimated.

\section{Perfusion scintigraphy}

${ }^{99 \mathrm{~m}} \mathrm{Tc}$-labelled macro-aggregated human albumin particles (MAA) are injected into a peripheral vein leading to pulmonary micro-embolisation. To obtain uniform distribution of activity reflecting regional perfusion, a minimum of 60,000 particles is required [34]. In the usual non-pregnant adult, normally about 400,000 labelled particles are injected.
A reduction in the number of particles administered to between 100,000 and 200,000 is recommended in patients with known pulmonary hypertension, right to left heart shunt or after a single lung transplantation [35]. ${ }^{99 \mathrm{~m}} \mathrm{Tc}$ is excreted via the kidneys and while in the bladder it will contribute to the fetal dose. The biological half-life of pertechnetate ${ }^{99 \mathrm{~m}} \mathrm{Tc}$ is $55-108 \mathrm{~min}$ [36]. See Table 2 for an overview of the studies discussed.

Using a low-dose perfusion protocol of $40 \mathrm{MBq}{ }^{99 \mathrm{~m}} \mathrm{Tc}$ MAA, Nijkeuter et al. [24] published a fetal radiation dose of $0.11-0.2 \mathrm{mSv}$; the term of pregnancy was not further specified. Again, as in their fetal dosimetry for $\mathrm{CT}$, the calculations were based on the assumption that the amount of radiation absorbed by the fetus was equal to that absorbed by the uterus of a non-pregnant woman.

Cook and Kyriou [28] reported a fetal dose of $0.12 \mathrm{mGy}$ for perfusion scintigraphy, also using low-dose perfusion imaging (50 MBq ${ }^{99 \mathrm{~m}} \mathrm{Tc}$ ); neither further technical details nor term of pregnancy were further specified.

In publication 84, the ICRP published fetal dose estimations for early and late pregnancy using $200 \mathrm{MBq}$ ${ }^{99 \mathrm{~m}} \mathrm{Tc}$ for the calculations [29]. A fetal dose of 0.4$0.6 \mathrm{mGy}$ was estimated in early pregnancy, whilst in late pregnancy a dose of $0.8 \mathrm{mGy}$ was reported.

Mean fetal doses of $0.21-0.3 \mathrm{mGy}$ in early pregnancy were described by Hurwitz et al. [21] using $74 \mathrm{MBq}$ of ${ }^{99 \mathrm{~m}}$ Tc-MAA.

Huda [26] reported fetal doses of $0.9 \mathrm{mGy}$ for $370 \mathrm{MBq}$ ${ }^{99 \mathrm{~m}}$ Tc-MAA perfusion imaging, based on old numbers from the ICRP [37].

Russel et al. [38] published mean fetal doses for different stages of pregnancy. Using $200 \mathrm{MBq}{ }^{99 \mathrm{~m}} \mathrm{Tc}-\mathrm{MAA}$ they published $0.6 \mathrm{mGy}$ for the first trimester, $0.75 \mathrm{mGy}$ for the second trimester and $0.6 \mathrm{mGy}$ for the third trimester.

The NRPB published fetal doses of $0.2 \mathrm{mGy}$ for perfusion imaging with ${ }^{99 \mathrm{~m}}$ Tc-MAA [30]. No technical details or trimester were specified.

\section{Ventilation scintigraphy}

The choice of radiopharmaceuticals for the ventilation portion of the lung imaging can also affect fetal dose. A ventilation study can be performed using either noble gases, such as ${ }^{133} \mathrm{Xe}$ gas and ${ }^{81 \mathrm{~m}} \mathrm{Kr}$, or aerosolised ${ }^{99 \mathrm{~m}} \mathrm{Tc}$ diethylenetriaminepentaacetic acid (DTPA) and Technegas. Using ${ }^{133} \mathrm{Xe}$ results in a very low embryo dose $(<0.01 \mathrm{mGy})$. The inhalation of approximately $110 \mathrm{MBq}$ ${ }^{99 \mathrm{~m}} \mathrm{Tc}$ DTPA will result in an embryo dose of about $0.9 \mathrm{mGy}$ [37]. It is absorbed through the lung and excreted via the kidneys, and while in the bladder it will contribute to fetal dose. The biological half-life of ${ }^{99 \mathrm{~m}} \mathrm{Tc}$-Technegas (an ultra-fine suspension of carbon nano-particles labelled with technetium) is $135 \mathrm{~h} \mathrm{[39].}$ 
Table 2 Studies reporting fetal doses for perfusion scintigraphy in suspected PE ( $n f s$ not further specified)

\begin{tabular}{lllll}
\hline Author & \multicolumn{2}{l}{ Fetal dose (mGy) } & & \\
\cline { 2 - 5 } & 1st trimester & 2nd trimester & 3rd trimester & ${ }^{99 m}$ Tc-MAA \\
\hline Nijkeuter 2004 [24] & $0.11-0.2$ & - & - & $40 \mathrm{MBq}$ \\
Cook 2005 [28] & 0.12 & trimester nfs & - & $50 \mathrm{MBq}$ \\
Hurwitz 2006 [21] & $0.21-0.3$ & - & - & $74 \mathrm{MBq}$ \\
ICRP 2000 [29] & $0.4-0.6$ & - & 0.8 & $200 \mathrm{MBq}$ \\
Russel 1997 [38] & 0.6 & 0.75 & 0.6 & $200 \mathrm{MBq}$ \\
\hline
\end{tabular}

In the case of suspected $\mathrm{PE}$, the perfusion imaging can be performed first, and if it is normal, ventilation imaging may not be needed at all. Therefore, ventilation imaging in pregnancy should only be performed in exceptional cases and not using ${ }^{99 \mathrm{~m}} \mathrm{Tc}$-aerosols to minimise fetal radiation exposure. Even in the non-pregnant population the diagnostic value of perfusion imaging only combined with chest radiography seems underestimated. Recent publications show that a turn back to older imaging algorithms using chest radiography and perfusion scintigraphy provide diagnostic accuracy similar to CTA [40]. See Table 3 for an overview of the studies discussed.

Nijkeuter et al. [24] measured fetal dose using $600 \mathrm{MBq}$ ${ }^{81 \mathrm{~m}} \mathrm{Kr}$-aerosol gas in a 2-min rebreathing protocol, resulting in a dose of $0.0001 \mathrm{mGy}$.

The ICRP published dose estimates for fetal radiation using $40 \mathrm{MBq}^{99 \mathrm{~m}} \mathrm{Tc}$-aerosol. Fetal dose was estimated to be $0.1-0.3 \mathrm{mGy}$ in early and $0.1 \mathrm{mGy}$ in late pregnancy [29].

Again using $40 \mathrm{MBq}{ }^{99 m}$ Tc-DTPA aerosol, Russel et al. [38] reported a fetal dose of $0.17 \mathrm{mGy}$ in the first trimester, $0.092 \mathrm{mGy}$ in the second trimester and $0.12 \mathrm{mGy}$ in the third trimester of pregnancy. Using ${ }^{133} \mathrm{Xe}$ rebreathing protocols resulted in $0.000015-0.000048 \mathrm{mGy}$ fetal radiation.

The fetal radiation dose absorbed by maternal ventilation imaging was calculated, based on the data of Hurwitz et al. [21], as $0.15 \mathrm{mGy}$ in early pregnancy and $0.02 \mathrm{mGy}$ in late pregnancy with 5-min maternal rebreathing of $370 \mathrm{MBq}$ ${ }^{133} \mathrm{Xe}$. Another protocol using $740 \mathrm{MBq}{ }^{133}$ Xe breath-hold without maternal rebreathing resulted in $0.04 \mathrm{mGy}$ in early and $0.01 \mathrm{mGy}$ in late pregnancy.

The NRPB published fetal doses of up to $1.5 \mathrm{mGy}$ for ventilation imaging using ${ }^{99 \mathrm{~m}} \mathrm{Tc}$-aerosols [30].

\section{Discussion}

Direct dose measurements or calculations/estimates of fetal dose in chest CT are difficult to perform and in the literature a variety of doses is reported, most probably due to the background that fetal dose in chest CT is dependent on the position and size of the fetus as well as on the trimester of pregnancy [22]. A recent article published by the American College of Radiology points out that dose calculation is a very complex issue because absorption in each organ is variable from patient to patient [41].

Whether scintigraphy or CTA should be the preferred imaging technique remains a controversially debated topic. Already the PIOPED I trial showed that more than $60 \%$ of $\mathrm{V} / \mathrm{Q}$ imaging is non-diagnostic and additional diagnostic studies might be pursued, as the probability of PE is still considerable. This may result in delay and more radiation exposure, which is not desirable in pregnancy [31, 42]. However, a substantial reduction in the proportion of nondiagnostic scintigraphy studies has been achieved since PIOPED $[43,44]$. In a recent publication, lung scintigraphy had a lower diagnostic inadequacy rate than CTA in

Table 3 Studies reporting fetal doses for ventilation scintigraphy ( $n f s$ not further specified)

\begin{tabular}{|c|c|c|c|c|c|c|}
\hline \multirow[t]{2}{*}{ Author } & \multicolumn{6}{|l|}{ Fetal dose (mGy) } \\
\hline & 1st trimester & 2nd trimester & 3rd trimester & Activity & Radionuclide & Specials \\
\hline Nijkeuter 2004 [24] & 0.0001 & Trimester nfs & & $600 \mathrm{MBq}$ & ${ }^{81 \mathrm{~m}} \mathrm{Kr}$-aerosol gas & 2 min rebreathing \\
\hline Hurwitz 2006 [21] & 0.15 & - & 0.02 & $370 \mathrm{MBq}$ & ${ }^{133} \mathrm{Xe}$-aerosol gas & 5 min rebreathing \\
\hline Hurwitz 2006 [21] & 0.04 & - & 0.01 & $740 \mathrm{MBq}$ & ${ }^{133} \mathrm{Xe}$-aerosol gas & Breathhold \\
\hline Russel 1997 [38] & $0.000015-0.000048$ & Trimester nfs & & $\mathrm{nfs}$ & ${ }^{133} \mathrm{Xe}$-aerosol gas & Rebreathing \\
\hline NRPB 1993 [30] & 1.5 & Trimester nfs & & $\mathrm{nfs}$ & ${ }^{99 m}$ Tc-aerosol & - \\
\hline ICRP 2000 [29] & $0.1-0.3$ & - & 0.1 & $40 \mathrm{MBq}$ & ${ }^{99 m}$ Tc-aerosol & - \\
\hline Russel 1997 [38] & 0.17 & 0.092 & 0.12 & $40 \mathrm{MBq}$ & ${ }^{99 m}$ Tc-aerosol & - \\
\hline
\end{tabular}


pregnant patients [45], probably due to a transient interruption of contrast material by unopacified blood from the inferior vena cava in the CTA studies. Still, today, the standard assumption in radiological practice remains that work-up of suspected LE in pregnant women should be performed by scintigraphy because of the lower quantity of radiation administered to the fetus.

\section{International recommendations}

In the literature, even international recommendations remain unclear as to whether to use scintigraphy or CTA for pregnant patients.

Several national and international societies, such as the Fleischner Society or the PIOPED II investigators, recommend D-dimer assessment and the use of ultrasound instead of $\mathrm{CT}$ or scintigraphy as the first-line imaging test for the evaluation of pregnant patients [20, 46, 47]. According to the guidelines of the German Society of Radiology, CT is the preferred technique because of the lower radiation dose to the uterus, but clinical risk assessment and combined Ddimer testing is recommended before imaging strategies are attempted [46].

If imaging with ionising radiation is necessary, both CT angiography and ventilation-perfusion lung imaging can provide useful information; however, $\mathrm{CT}$ might be the preferred technique because of exposure of the conceptus to lower doses [48].

The British Thoracic Society guidelines give no specific recommendations for imaging pregnant patients for suspected PE, but declare $\mathrm{CT}$ to be the initial lung imaging technique in the general patient. Isotope lung imaging may be considered if several quality criteria are fulfilled [47].

In a statement by the Fleischner Society, strategies for pregnant patients were briefly discussed, but with no direct recommendations being given [20]. However, even among the PIOPED II investigators the discussion remained controversial. Most of the PIOPED II investigators recommended D-dimer assessment and ultrasound before perfusion scintigraphy for suspected pulmonary embolisms in pregnant patients $[15,48]$.

Fetal radiation dose

Many methods for the estimation of fetal dose in pregnant patients undergoing CT examinations assumed early term pregnancy in a single-size patient model with an average, uniform maternal anatomy. These dose estimates did not take into account maternal anatomy variances, natural variations, such as fetal presentation, and gestational age. Differences in these attributes can cause failures in estimation of fetal dose of up to $100 \%$ at radiological examinations $[49,50]$. The fetal dose will increase during pregnancy both as the fetus grows in size and as it moves closer to the imaging volume [23].

In the first trimester of pregnancy, the absorbed dose to the uterus may be used as a substitute for the absorbed dose to the embryo. Similarly, the absorbed dose to the fetus from radioactive substances without placental transfer is expected to be within the same range as the dose to the uterus. In the case of radioactive substances with placental transfer, the absorbed dose to organs and tissues of the mother may, as a first approximation, be taken as representative of the absorbed dose to the corresponding organs and tissues of the fetus [51].

In CTA, the fetus is only exposed to scatter radiation assuming appropriate planning of the examination. Inadvertent irradiation of a fetus most frequently occurs during the early post-conception period, when the woman is unaware of her pregnancy. In early pregnancy, the distance from the directly irradiated region at the base of the lung to the location of the embryo is generally at least $20 \mathrm{~cm}$, where the scattered radiation level would be no more than $1 \%$ of the chest dose [25].

The risks of causing a range of different radiation effects depend on the gestational age [52]. In fact, in the preimplantation embryo, there is no measurable risk of malformation regardless of the amount of radiation exposure, and the greatest concern is death of the embryo.

Within the first 2 weeks of embryonic age, there is a $2 \%$ risk of the blastocyst failing to implant, causing death of the embryo when the irradiation dose is greater than $0.1 \mathrm{~Gy}$. If the embryo survives there is likely to be no increased risk $[53,54]$.

During the first two trimesters the fetus is most susceptible to deterministic teratogenic effects, but it is assumed that the fetus is not at risk if the irradiation dose does not exceed a threshold dose [55]. None of the studies analysed explicitly reported fetal doses when applying dose reduction measures such as lead shielding or automatic exposure control (AEC), only relative changes in percentage. Therefore, the absolute fetal dose using recent imaging technology including dose reduction strategies must be estimated to be lower than those reported.

Meeting the threshold for inducing deterministic effects as malformations or reduction in intelligence is not likely with the expected doses in $\mathrm{CT}$ or in V/P imaging either; thus, the most important consideration is induction of childhood cancer. As in all examinations in radiology, there is always a stochastic risk of carcinogenesis, and also in the fetus after in utero irradiation, regardless of the dose. Despite the fact that fetal radiation dose for both CTA and scintigraphy examinations in suspected pregnancy-related PE must be seen to be very low, further reduction must be aimed at during pregnancy. The risks of low-level radiation are difficult to quantify and the risk of malignancy is 
known to be increased in persons that have been exposed to radiation in utero [56]. However, many effects of radiationinduced childhood cancer still remain controversial [57].

The fetal doses in CTA reported in the literature are well below the 100-mGy threshold reported to be relevant for deterministic effects [29]. The fetal dose of $50 \mathrm{mGy}$ is considered as the limit below which there is no harm from deterministic effects and the risk of stochastic effects is $<1 \%$ [58]. There is no evidence that the pattern of cancer induction varies with gestational age and the risk is likely to be constant over the entire pregnancy [59]. The number of excess malignancy cases up to the age of 15 years following irradiation in utero is considered to be 1 in 16,000 per $\mathrm{mSv}$ [59]. This equates to an additional risk of malignancy of 1 in 560,000 following half-dose perfusion scintigraphy and 1 in $1,000,000$ after CT pulmonary angiography [28]. Other publications report the excess risk of the induction of childhood cancer to be 1 in 33,000 per mGy [52]. In addition, the excess relative risk of developing childhood cancer has been estimated to be approximately 0.28 at $1.0 \mathrm{mGy}$ in the first trimester, 0.03 at $1.0 \mathrm{mGy}$ in the third trimester, and overall 0.037 at 1.0 mGy during pregnancy [59]. A recent paper reports the probability of giving birth to a healthy baby decreases by only $0.5 \%$ even when performing routine dose level biphasic CT of the abdomen [60]. The fetal dose level for chest CT is far beyond these estimated dose values [60]. Fetal threshold doses and risks in different gestational periods have been outlined extensively [61].

The natural background radiation dose to the fetus during pregnancy is approximately $1 \mathrm{mGy}$ [57]. By comparison, exposure of at least $100 \mathrm{mGy}$ is necessary before pregnancy termination is considered [42].

In chest $\mathrm{CT}$, the fetal doses reported for specified trimesters range between 0.003 and $0.47 \mathrm{mGy}$ in the first trimester [21, 23, 24, 26]. Two studies determined fetal dose measurements in the second trimester of pregnancy to be $0.0079-0.66 \mathrm{mGy}[21,23]$. A variance of a factor of about 80 could be observed. For the third trimester, a wide variety of fetal doses was reported, such as $0.051-1.2 \mathrm{mGy}$, differing by a factor of nearly $30[22,23,27]$.

Similarly the high fetal doses reported by Hurwitz et al. [21] must be reviewed critically. These were the only authors using a tube current of $140 \mathrm{kV}$, together with a high tube current product of $380 \mathrm{mAs}$ for the imaging of pregnant patients. Therefore, we attribute the high reported fetal dose of $0.24-0.66 \mathrm{mGy}$ in the first trimester to the selection of technical parameters for both tube current and tube voltage, which were above recent recommendations.

Conversely, Winer-Muram et al. [23] calculated very low fetal doses. Fetal and uterus dimensions were precisely measured in 23 pregnant women, who formed the basis for the dose calculations. The imaging parameters used were reasonable $(120 \mathrm{kV}, 100 \mathrm{mAs}$, pitch 1$)$, but the imaging distance was described as $11 \mathrm{~cm}$ from just inferior to the xiphoid process to the aortic arch. We do not estimate this image length to be sufficient to rule out maternal PE properly. Using commercial dosimetry tools [62], the image length to cover the whole lungs is around $24 \mathrm{~cm}$. Even reducing the basal image length, as has been proposed in the literature, results in an image length of $19 \mathrm{~cm}$ [22]. The amount of fetal radiation in the calculations might not reflect the scatter radiation in routine chest CT examinations for suspected PE. Therefore, we think that the fetal doses reported from Winer-Muram and co-workers cannot be associated with an image length covering the whole lungs.

The fetal doses reported for perfusion scintigraphy are all very consistent for all trimesters [21, 24, 28, 29, 38]. Reducing administered activity of ${ }^{99 \mathrm{~m}} \mathrm{Tc}-\mathrm{MAA}$ to $25 \%$ equally reduces the fetal dose calculated. Therefore, possible dose reduction can be performed easily and straightforwardly. However, a minimum of 60,000 particles is required [34]; thus, there are clearly limits in further fetal dose reduction.

Exclusion of the CTA data discussed above leaves us with only the data published by Nijkeuter et al. [24] and, therefore, CTA mean fetal doses of $0.013 \mathrm{mGy}$ in the first trimester using recent multislice technology. For the second trimester no data remain to be discussed. However, as fetal size increases, the dose must be assumed to be somewhere between the first and third trimester data. In the third trimester at least two studies remain to give an average fetal dose of 0.06-0.14 mGy [22, 26]. These data are consistent with the average doses reported for the whole pregnancy, which range between 0.01 and $0.06 \mathrm{mGy}$ [28-30].

Taking into account recent recommendations to perform perfusion scintigraphy with "half dose" activity, perfusion scintigraphy resulted in mean fetal doses of $0.177 \mathrm{mGy}$ (SD 0.095) for all trimesters based on the data analysed [24, 28].

Ventilation imaging can and should be avoided in pregnant women if perfusion imaging is within normal limits. The fetal radiation dose strongly depends on the substances used for inhalation. Noble gases such as ${ }^{81 \mathrm{~m}} \mathrm{Kr}$ and ${ }^{133} \mathrm{Xe}$ should be preferred over ${ }^{99 \mathrm{~m}} \mathrm{Tc}$-aerosols.

There is a reported overall excess relative risk of childhood cancer of 0.037 at $1 \mathrm{mGy}$ of fetal radiation during pregnancy [59]. In the mother, a risk of radiationinduced breast cancer of $0.005 \%$ for $1 \mathrm{mSv}$ of radiation is reported [42]. This reflects an absorbed solitary dose of 8.3 mGy calculated with the new tissue weighting factors for breasts that have been raised from 0.05 to 0.12 [63]. These calculations were performed using a recent imaging protocol to detect suspected PE using a 16-row system and commercial dosimetry software [62, 64]. For the CT protocol applied by Nijkeuter et al. [59] we calculated a 
breast dose of $11 \mathrm{mGy}$ or $1.32 \mathrm{mSv}$ respectively, associated with a $0.017 \mathrm{mGy}$ fetal dose in the first trimester. Therefore the maternal breast cancer risk was 0.0066 and the fetal excess risk of childhood cancer around 0.000476 in early pregnancy using the numbers of the ICRP. Based on the numbers by Doshi et al. [22], we calculated for the same image length and late pregnancy a breast dose of $10 \mathrm{mGy}$ or $1.2 \mathrm{mSv}$ respectively, associated with $0.1 \mathrm{mGy}$ fetal dose. Therefore, the maternal breast cancer risk was 0.006 and the fetal excess risk of childhood cancer was around 0.003 .

Based on the data analysed for perfusion-only scintigraphy the absorbed breast dose is around $0.044 \mathrm{mSv}$ for lowdose perfusion scintigraphy $(74 \mathrm{MBq})$ and $0.12 \mathrm{mSv}$ for normal dose scintigraphy (200 MBq). This leads to a maternal breast cancer risk of 0.0002-0.0006 (74$200 \mathrm{MBq}$ ). For early/late pregnancy a fetal dose of $0.11-$ $0.6 \mathrm{mGy}\left(74-200 \mathrm{MBq}{ }^{99 \mathrm{~m}} \mathrm{Tc}-\mathrm{MAA}\right) / 0.8 \mathrm{mGy}(200 \mathrm{MBq}$ ${ }^{99 m}$ Tc-MAA) could be observed. This results in a fetal excess risk of childhood cancer of around 0.0031-0.17 (74$200 \mathrm{MBq}{ }^{99 m}$ Tc-MAA) in early and 0.024 (200 MBq ${ }^{99 \mathrm{~m}}$ Tc-MAA) in late pregnancy.

\section{Abdominal lead shielding}

Maternal abdominal lead shielding has been reported to be an effective way of reducing fetal scatter radiation [22]. Circumferential lead covering yields in a double dose reduction to the fetus in comparison with anterior shielding only [65]. The contribution to the fetal dose of secondary internally scattered radiation increases with increasing lead thickness but reaches a plateau at a certain thickness of lead. The component resulting from secondary internally scattered radiation is much smaller in magnitude than the external and primary internal scatter radiation. The primary internal scatter radiation can be seen as independent of the thickness of lead used for shielding [66]. Of course, allowing the shielding to reach the direct radiation exposure field must be carefully avoided because the automatic tube current regulation would immediately cause a tremendous increase in the applied dose.

The effectiveness of performing imaging with internal as opposed to external abdominal shielding has been demonstrated in a phantom study using orally administered barium sulphate to protect the fetus [67].

Tube current

Technological advantages such as automatic tube current modulation tools should be used in both pregnant and nonpregnant patients.

Doshi et al. [22] reported an overall dose reduction of $10 \%$ using automatic tube current modulation in their simulation study. Depending on the image noise level other studies even yielded dose reduction of $32-66 \%$ using AEC [68-71].

Image length

As could be shown in phantom measurements, a reduction of the image length is a valuable way of reducing the dose to the fetus. Sparing the lung bases yields significant fetal dose reduction $[65,72]$. Emboli that could be missed on CT sparing the lung bases are to be found in a subsegmental distribution. The clinical significance of subsegmental pulmonary embolism is still debated controversially. It has been suggested that emboli in the subsegmental pulmonary arteries do not contribute substantially to morbidity and mortality, and taking into account the uncertainty regarding the necessity of treating patients with isolated subsegmental $\mathrm{PE}$, the clinical impact of the depiction of subsegmental clots still remains unclear [73-75]. Based on this assumption protocols can be used covering not the whole lung but sparing the lung bases to maximise the distance between the fetus and the edge of the image $[65,76]$.

\section{Maternal breast tissue}

Although still debated controversially, radiation dose to the breast tissue seems to be of critical importance, especially in girls and young women. Just recently, tissue weighting factors for breast tissue have more than doubled, taking into account recent research implying possible elevated radiosensitivity [63]. However, CT radiation risks for breast tissue still remain unclear [77].

In the case of chest CT, it is clear that the maternal organ at greatest risk is the female breast. In particular, the proliferating breast tissue in pregnancy seems likely to be at increased risk. Breast doses have been estimated to be 20 $60 \mathrm{mGy}$ for a CT examination performed for pulmonary embolism [78-80]. For these breast doses reported, the authors used technical parameters that should not be applied in pregnancy, such as tube voltage of $140 \mathrm{kV}[79$, $80]$ and a tube current product of a maximum of $304 \mathrm{mAs}$ [80]. Applying a reasonable tube voltage of $120 \mathrm{kV}$ results in breast doses of a maximum of $26 \mathrm{mGy}$ based on the technical parameters reported.

Conversely, injection of ${ }^{99} \mathrm{~m}$ Tc-MAA results in an absorbed maternal breast dose of $0.005 \mathrm{mGy} / \mathrm{MBq}$ per unit of activity administered [81]. Dose-reduced perfusion imaging therefore results in an absorbed breast dose of 0.2-0.37 mGy, assuming administered activity of 40$74 \mathrm{MBq}$. Normal dose perfusion imaging with $200 \mathrm{MBq}$ ${ }^{99 \mathrm{~m}}$ Tc-MAA accounts for $1 \mathrm{mGy}$ of breast radiation.

For breast cancer, an associated risk of $0.005 \%$ has been reported for $1 \mathrm{mSv}$ of radiation exposure. Considering the 
baseline risk that approximately $23 \%$ of the population will develop cancer at some point in their lives, the increased risk due to CT is very small [42]. Also, some authors try to push the discussion back to reason and point out that many clinicians underestimate the risk of radiation, but equally others massively overestimate the risk [82].

Breast bismuth shields have been evaluated in the literature to reduce the dose to the female/maternal breast and are reported to be an effective measure [60, 83]. However, their radiological value still seems to be controversial, as other authors do not recommend the use of breast shielding because of the impact on image noise. If an increase in image noise is accepted, reduction of the tube current is proposed as an easier and more efficient dose reduction strategy [84]. Recent articles also promote the use of AEC for significant breast dose reduction [85].

\section{Iodinated contrast media}

Iodinated contrast media are necessary when performing CTA for suspected PE and both the mother and the fetus are exposed to intravenous iodinated contrast medium. These may traverse the placenta and enter the fetal blood, then excreted by the kidneys into the bladder and actually reach the amniotic fluid. When the amniotic fluid is swallowed it enters the gut. Depression of fetal thyroid function is the chief potential harmful effect of iodinated contrast media within the fetus [86]. There is still a lack of scientific data on the amount of free iodine possibly traversing the placenta and its side effects and consequences for the fetus. When the mother has received iodinated contrast media, the thyroid function of the newborn should be checked during the first week $[13,86]$.

When performing CTA in pregnancy, deterioration in pulmonary vessel enhancement has been reported, probably due to hyperdynamic circulation with increased cardiac output, increased plasma volume, increased body weight and the more pronounced effects of a Valsalva manoeuvre $[45,87]$. Use of bolus triggering protocols in the imaging planning should be preferred over fixed delays to overcome possible contrast limitations in pregnancy. Further contrast optimisation has been reported to be achieved with tube voltage reduction, as the effective energy of the X-ray beam approaches the absorption k-edge of iodine [72, 87-90]. In addition substantial dose reduction can be assumed for both the maternal breasts and the fetus.

\section{Value of chest radiography}

A preliminary chest radiograph remains important in all pregnant patients for the exclusion of alternative readily diagnosable conditions and maybe to aid in the interpretation of subsequent tests. The chest radiograph also plays an important but often overlooked role in triaging subsequent imaging tests (i.e. scintigraphy or MDCT). For this purpose a single plane radiograph is absolutely sufficient. It has been shown that the presence of any abnormality on the initial chest radiograph decreases the utility of scintigraphy $[91,92]$. The approximate fetal dose from a single plane chest radiograph is reported to be $<0.01 \mathrm{mGy}[21,29]$. However, in scintigraphy examinations easy further measures can also provide dose reduction and increase diagnostic accuracy.

\section{Voiding after scintigraphy}

Radionuclides in maternal tissues contribute to fetal dose. In the case of radiopharmaceuticals that are rapidly eliminated by the maternal kidneys such as ${ }^{99 \mathrm{~m}} \mathrm{Tc}$, the urinary bladder may be acting as a reservoir and is a major source of fetal irradiation. Maternal hydration and frequent voiding can reduce the fetal dose after the administration of a number of radiopharmaceuticals, in this context especially of ${ }^{99 \mathrm{~m}} \mathrm{Tc}$ [29].

\section{Value of SPECT scintigraphy}

Single photon emission computed tomography (SPECT) further increases the diagnostic accuracy of the scintigraphy images using two main methods [32]. One method sums projections over a limited angular range, while another uses reconstructed SPECT data projected through an attenuation map to generate count-rich reprojected planar images [93]. Bajc et al. [94] identified 53\% more mismatched regions with SPECT. In a study by Collart et al. [95], V/P increased the specificity for PE from $78 \%$ to $96 \%$ at similar sensitivities to CT. Reinartz et al. [96] found a sensitivity and specificity of 0.76 and 0.85 , respectively, with $\mathrm{V} / \mathrm{P}_{\text {PLANAR }}$ compared with 0.97 and 0.91 with $\mathrm{V} / \mathrm{P}_{\text {SPECT }}$.

In summary, CTA for PE has the advantage that the fetus is not directly exposed, but only to scatter radiation. The estimated radiation exposure is low for CT when the fetus is outside the field of view.

In perfusion scintigraphy, the need for intravenous injection of the radionuclide tracer will lead to perfusiondependent and bladder-reservoir-related direct fetal exposure. In particular, in the first trimester fetal exposure with CTA is therefore lower than exposure with perfusion scintigraphy, even if a half-dose scintigraphic technique is used [24, 28].

For chest CT, most of the studies cited reported mean fetal dose estimates to be lower by a factor of 10 than the comparable dose-reduced perfusion scintigraphy protocols. These estimates did not take into account the different dose reduction measures possible in CTA. Thus, fetal dose must 
be estimated as being substantially lower for chest CT than for the scintigraphy pathway.

Based on the data analysed, there is an increased risk of childhood cancer induction in early pregnancy when using perfusion scintigraphy of a factor of about 7 ; even in late pregnancy the risk is still increased by a factor of about 8 compared with the estimated risk calculated for CTA.

The maternal breast cancer risk was calculated to be increased about tenfold in CTA compared with perfusion scintigraphy over the whole pregnancy.

These risk estimates are applied for normal dose perfusion scintigraphy and CTA without dose reduction measures.

With CTA, dose reduction measures such as abdominal lead shielding, reduced tube current, AEC, reduced tube voltage and reduced image length the maternal and especially the fetal dose can be expected to be further reduced substantially.

In perfusion scintigraphy, dose reduction is directly related to the activity administered. However, the breast dose reduction that can be estimated by reducing the activity administered in perfusion scintigraphy seems to be far more than can be expected from CT dose reduction measures.

\section{Conclusion}

According to the ICRP, almost always, if a diagnostic radiology examination is medically indicated, the risk to the mother of not carrying out the procedure is greater than the risk of potential harm to the fetus. Radiation doses resulting from most diagnostic procedures present no substantial risk of causing fetal death, malformation, or impairment of mental development [29].

As CTA allows a much wider variety of pathological conditions to be depicted and as the fetal dose is substantially lower than with the use of scintigraphy, it is reasonable for CTA to be performed in suspected pregnancy-related PE. However, the breast dose absorbed by the mother is higher than with the use of scintigraphy.

It is reasonable to reserve scintigraphy for patients with normal chest radiographs without any remarkable findings and no history of asthma or chronic lung disease. Recent imaging technology and $\mathrm{CT}$ dose reduction strategies should be applied when imaging pregnant women for suspected PE. Ventilation scintigraphy using ${ }^{99 \mathrm{~m}} \mathrm{Tc}$-aerosols should not be performed in pregnancy.

Radiation in pregnancy should be avoided whenever possible. The risks and benefits of radiation exposure both to mother and fetus must be weighed up when deciding imaging strategies in pregnancy.

Overall, the risks to the conceptus are negligible at doses such as those involved in CT and scintigraphy for PE.

\section{References}

1. James AH, Jamison MG, Brancazio LR, Myers ER (2006) Venous thromboembolism during pregnancy and the postpartum period: incidence, risk factors, and mortality. Am J Obstet Gynecol 194:1311-1315

2. Pabinger I, Grafenhofer H, Kaider A et al (2005) Risk of pregnancyassociated recurrent venous thromboembolism in women with a history of venous thrombosis. J Thromb Haemost 3:949-954

3. Pabinger I, Grafenhofer H (2002) Thrombosis during pregnancy: risk factors, diagnosis and treatment. Pathophysiol Haemost Thromb 32:322-324

4. Kuklina EV, Meikle SF, Jamieson DJ et al (2009) Severe obstetric morbidity in the United States: 1998-2005. Obstet Gynecol 113:293-299

5. Matthews S (2006) Short communication: imaging pulmonary embolism in pregnancy: what is the most appropriate imaging protocol? Br J Radiol 79:441-444

6. Wells PS, Anderson DR, Rodger M et al (2001) Excluding pulmonary embolism at the bedside without diagnostic imaging: management of patients with suspected pulmonary embolism presenting to the emergency department by using a simple clinical model and d-dimer. Ann Intern Med 135:98-107

7. Morse M (2004) Establishing a normal range for D-dimer levels through pregnancy to aid in the diagnosis of pulmonary embolism and deep vein thrombosis. J Thromb Haemost 2:1202-1204

8. Onishi H, Kaniyu K, Iwashita M, Tanaka A, Watanabe $T$ (2007) Fibrin monomer complex in normal pregnant women: a potential thrombotic marker in pregnancy. Ann Clin Biochem 44:449-454

9. Pahade JK, Litmanovich D, Pedrosa I, Romero J, Bankier AA, Boiselle PM (2009) Quality initiatives: imaging pregnant patients with suspected pulmonary embolism: what the radiologist needs to know. Radiographics 29:639-654

10. Safety and efficacy of computed tomography (CT): a broad perspective. www.msct.eu. 2005

11. Hall EJ, Brenner DJ (2008) Cancer risks from diagnostic radiology. Br J Radiol 81:362-378

12. Brenner DJ, Hall EJ (2007) Computed tomography-an increasing source of radiation exposure. $N$ Engl J Med 357:2277-2284

13. Siegmann KC, Heuschmid M, Claussen CD (2009) Diagnostic imaging during pregnancy. Dtsch Med Wochenschr 134:686-689

14. Groves AM, Yates SJ, Win T et al (2006) CT pulmonary angiography versus ventilation-perfusion scintigraphy in pregnancy: implications from a UK survey of doctors' knowledge of radiation exposure. Radiology 240:765-770

15. Stein PD, Woodard PK, Weg JG et al (2006) Diagnostic pathways in acute pulmonary embolism: recommendations of the PIOPED II investigators. Am J Med 119:1048-1055

16. Stein PD, Kayali F, Olson RE (2004) Trends in the use of diagnostic imaging in patients hospitalized with acute pulmonary embolism. Am J Cardiol 93:1316-1317

17. Schoepf UJ, Goldhaber SZ, Costello P (2004) Spiral computed tomography for acute pulmonary embolism. Circulation 109:2160-2167

18. Schoepf UJ, Costello P (2004) CT angiography for diagnosis of pulmonary embolism: state of the art. Radiology 230:329-337

19. Stein PD, Fowler SE, Goodman LR et al (2006) Multidetector computed tomography for acute pulmonary embolism. N Engl J Med 354:2317-2327

20. Remy-Jardin M, Pistolesi M, Goodman LR et al (2007) Management of suspected acute pulmonary embolism in the era of CT angiography: a statement from the Fleischner Society. Radiology 245:315-329 
21. Hurwitz LM, Yoshizumi T, Reiman RE et al (2006) Radiation dose to the fetus from body MDCT during early gestation. AJR Am J Roentgenol 186:871-876

22. Doshi SK, Negus IS, Oduko JM (2008) Fetal radiation dose from CT pulmonary angiography in late pregnancy: a phantom study. Br J Radiol 81:653-658

23. Winer-Muram HT, Boone JM, Brown HL, Jennings SG, Mabie WC, Lombardo GT (2002) Pulmonary embolism in pregnant patients: fetal radiation dose with helical CT. Radiology 224:487-492

24. Nijkeuter M, Geleijns J, De RA, Meinders AE, Huisman MV (2004) Diagnosing pulmonary embolism in pregnancy: rationalizing fetal radiation exposure in radiological procedures. $\mathrm{J}$ Thromb Haemost 2:1857-1858

25. Boone JM, Cooper VN III, Nemzek WR, McGahan JP, Seibert JA (2000) Monte Carlo assessment of computed tomography dose to tissue adjacent to the scanned volume. Med Phys 27:2393-2407

26. Huda W (2005) When a pregnant patient has a suspected pulmonary embolism, what are the typical embryo doses from a chest $\mathrm{CT}$ and a ventilation/perfusion study? Pediatr Radiol 35:452-453

27. Damilakis J, Perisinakis K, Voloudaki A, Gourtsoyiannis N (2000) Estimation of fetal radiation dose from computed tomography scanning in late pregnancy: depth-dose data from routine examinations. Invest Radiol 35:527-533

28. Cook JV, Kyriou J (2005) Radiation from CT and perfusion scanning in pregnancy. BMJ 331:350

29. ICRP (2000) Pregnancy and medical radiation. Ann ICRP 30:1-43

30. NRPB (1993) Diagnostic medical exposures: exposure to ionising radiation of pregnant women. Doc NRPB 4:5-14

31. Investigators TPIOPED (1990) Value of the ventilation/perfusion scan in acute pulmonary embolism. Results of the prospective investigation of pulmonary embolism diagnosis (PIOPED). JAMA 263:2753-2759

32. Reid JH, Coche EE, Inoue T et al (2009) Is the lung scan alive and well? Facts and controversies in defining the role of lung scintigraphy for the diagnosis of pulmonary embolism in the era of MDCT. Eur J Nucl Med Mol Imaging 36:505-521

33. Scarsbrook AF, Bradley KM, Gleeson FV (2007) Perfusion scintigraphy: diagnostic utility in pregnant women with suspected pulmonary embolic disease. Eur Radiol 17:2554-2560

34. Heck LL, Duley JW Jr (1974) Statistical considerations in lung imaging with 99mTc albumin particles. Radiology 113:675-679

35. Bajc M, Neilly JB, Miniati M, Schuemichen C, Meignan M, Jonson B (2009) EANM guidelines for ventilation/perfusion scintigraphy: Part 1. Pulmonary imaging with ventilation/perfusion single photon emission tomography. Eur J Nucl Med Mol Imaging 36:1356-1370

36. Strong JC, Agnew JE (1989) The particle size distribution of technegas and its influence on regional lung deposition. Nucl Med Commun 10:425-430

37. Radiation dose to patients from radiopharmaceuticals (1987) A report of a Task Group of Committee 2 of the International Commission on Radiological Protection. Ann ICRP 18:1-377

38. Russell JR, Stabin MG, Sparks RB, Watson E (1997) Radiation absorbed dose to the embryo/fetus from radiopharmaceuticals. Health Phys 73:756-769

39. Kawakami K, Iwamura A, Goto E et al (1990) Kinetics and clinical application of 99mTc-technegas. Kaku Igaku 27:725-733

40. Sostman HD, Miniati M, Gottschalk A, Matta F, Stein PD, Pistolesi M (2008) Sensitivity and specificity of perfusion scintigraphy combined with chest radiography for acute pulmonary embolism in PIOPED II. J Nucl Med 49:1741-1748

41. Amis ES Jr, Butler PF, Applegate KE et al (2007) American College of Radiology white paper on radiation dose in medicine. $\mathrm{J}$ Am Coll Radiol 4:272-284

42. Mallick S, Petkova D (2006) Investigating suspected pulmonary embolism during pregnancy. Respir Med 100:1682-1687
43. Barghouth G, Yersin B, Boubaker A, Doenz F, Schnyder P, Delaloye AB (2000) Combination of clinical and V/Q scan assessment for the diagnosis of pulmonary embolism: a 2-year outcome prospective study. Eur J Nucl Med 27:1280-1285

44. Bajc M, Albrechtsson U, Olsson CG, Olsson B, Jonson B (2002) Comparison of ventilation/perfusion scintigraphy and helical CT for diagnosis of pulmonary embolism; strategy using clinical data and ancillary findings. Clin Physiol Funct Imaging 22:392-397

45. Ridge CA, McDermott S, Freyne BJ, Brennan DJ, Collins CD, Skehan SJ (2009) Pulmonary embolism in pregnancy: comparison of pulmonary CT angiography and lung scintigraphy. AJR Am J Roentgenol 193:1223-1227

46. Deutsche Gesellschaft für Angiologie (2005) Diagnostik und Therapie der Bein- und Beckenvenenthrombose und der Lungenembolie. Phlebologie 34:47-64

47. British Thoracic Society Standards of Care Committee Pulmonary Embolism Guideline Development Group (2003) British Thoracic Society guidelines for the management of suspected acute pulmonary embolism. Thorax 58:470-483

48. Stein PD, Woodard PK, Weg JG et al (2007) Diagnostic pathways in acute pulmonary embolism: recommendations of the PIOPED II Investigators. Radiology 242:15-21

49. Osei EK, Faulkner K (1999) Fetal position and size data for dose estimation. Br J Radiol 72:363-370

50. Angel E, Wellnitz CV, Goodsitt MM et al (2008) Radiation dose to the fetus for pregnant patients undergoing multidetector CT imaging: Monte Carlo simulations estimating fetal dose for a range of gestational age and patient size. Radiology 249:220-227

51. ICRP (2008) Radiation dose to patients from radiopharmaceuticals. Addendum 3 to ICRP Publication 53. ICRP Publication 106. Approved by the Commission in October 2007. Ann ICRP 38:1197

52. National Radiological Protection Board RCoR (1998) Advice on exposure to ionising radiation during pregnancy. Diagnostic medical exposures. NRPB, Didcot

53. De Santis M, Cesari E, Nobili E, Straface G, Cavaliere AF, Caruso A (2007) Radiation effects on development. Birth Defects Res C Embryo Today 81:177-182

54. Bianca S (2005) Health risks of low-dose ionizing radiation in humans. Exp Biol Med (Maywood) 230:99-100

55. De Santis M, Di Gianantonio E, Straface G et al (2005) Ionizing radiations in pregnancy and teratogenesis: a review of literature. Reprod Toxicol 20:323-329

56. Preston DL, Cullings H, Suyama A et al (2008) Solid cancer incidence in atomic bomb survivors exposed in utero or as young children. J Natl Cancer Inst 100:428-436

57. Patel SJ, Reede DL, Katz DS, Subramaniam R, Amorosa JK (2007) Imaging the pregnant patient for nonobstetric conditions: algorithms and radiation dose considerations. Radiographics 27:1705-1722

58. NCRP (1994) Considerations Regarding the Unintended Radiation Exposure of the Embryo, Fetus or Nursing Child. Commentary No. 9. National Council on Radiation Protection and Measurements, Bethesda

59. Streffer C, Shore R, Konermann G et al (2003) Biological effects after prenatal irradiation (embryo and fetus). A report of the International Commission on Radiological Protection. Ann ICRP 33:5-206

60. McCollough CH, Primak AN, Braun N, Kofler J, Yu L, Christner J (2009) Strategies for reducing radiation dose in CT. Radiol Clin North Am 47:27-40

61. Steenvoorde P, Pauwels EK, Harding LK, Bourguignon M, Mariere B, Broerse JJ (1998) Diagnostic nuclear medicine and risk for the fetus. Eur J Nucl Med 25:193-199

62. ImPACT 2003. CT patient dosimetry Excel spreadsheet v0.99u (12/ 12/03). Available from home webpage of the ImPACT (Imaging 
Performance and Assessment of CT scanners) evaluation center of the DH Medical Devices Agency (http://www.impactscan.org). 2009

63. The 2007 Recommendations of the International Commission on Radiological Protection. ICRP publication 103. Ann ICRP 37:1332

64. Bongartz G, Golding SJ, Jurik A et al (2004) European guidelines for multislice computed tomography. www.msct.eu/cr_quality_criteria. htm

65. Kennedy EV, Iball GR, Brettle DS (2007) Investigation into the effects of lead shielding for fetal dose reduction in CT pulmonary angiography. Br J Radiol 80:631-638

66. Iball GR, Kennedy EV, Brettle DS (2008) Modelling the effect of lead and other materials for shielding of the fetus in CT pulmonary angiography. Br J Radiol 81:499-503

67. Yousefzadeh DK, Ward MB, Reft C (2006) Internal barium shielding to minimize fetal irradiation in spiral chest CT: a phantom simulation experiment. Radiology 239:751-758

68. Mulkens TH, Daineffe S, De WR et al (2007) Urinary stone disease: comparison of standard-dose and low-dose with 4D MDCT tube current modulation. AJR Am J Roentgenol 188:553-562

69. Kalra MK, Maher MM, D’Souza RV et al (2005) Detection of urinary tract stones at low-radiation-dose $\mathrm{CT}$ with z-axis automatic tube current modulation: phantom and clinical studies. Radiology 235:523-529

70. Mulkens TH, Bellinck P, Baeyaert M et al (2005) Use of an automatic exposure control mechanism for dose optimization in multi-detector row CT examinations: clinical evaluation. Radiology $237: 213-223$

71. Soderberg M, Gunnarsson M (2010) Automatic exposure control in computed tomography - an evaluation of systems from different manufacturers. Acta Radiol 51:625-634

72. Litmanovich D, Boiselle PM, Bankier AA, Kataoka ML, Pianykh O, Raptopoulos V (2009) Dose reduction in computed tomographic angiography of pregnant patients with suspected acute pulmonary embolism. J Comput Assist Tomogr 33:961-966

73. Remy-Jardin M, Remy J, Wattinne L, Giraud F (1992) Central pulmonary thromboembolism: diagnosis with spiral volumetric CT with the single-breath-hold technique-comparison with pulmonary angiography. Radiology 185:381-387

74. Dorffler-Melly J, Amann-Vesti B (2007) Diagnosis and treatment of acute pulmonary embolism. Herz 32:35-41

75. Eyer BA, Goodman LR, Washington L (2005) Clinicians' response to radiologists'reports of isolated subsegmental pulmonary embolism or inconclusive interpretation of pulmonary embolism using MDCT. AJR Am J Roentgenol 184:623-628

76. Howling SJ, Hansell DM (2000) Spiral computed tomography for pulmonary embolism. Hosp Med 61:41-45

77. John EM, Phipps AI, Knight JA et al (2007) Medical radiation exposure and breast cancer risk: findings from the Breast Cancer Family Registry. Int J Cancer 121:386-394

78. Mettler FA Jr, Huda W, Yoshizumi TT, Mahesh M (2008) Effective doses in radiology and diagnostic nuclear medicine: a catalog. Radiology 248:254-263

79. Parker MS, Hui FK, Camacho MA, Chung JK, Broga DW, Sethi NN (2005) Female breast radiation exposure during CT pulmonary angiography. AJR Am J Roentgenol 185:1228-1233

80. Hurwitz LM, Yoshizumi TT, Reiman RE et al (2006) Radiation dose to the female breast from 16-MDCT body protocols. AJR Am J Roentgenol 186:1718-1722
81. Radiation dose to patients from radiopharmaceuticals (addendum 2 to ICRP publication 53). Ann ICRP 1998; 28:1-126

82. Allen C, Demetriades T (2006) Radiation risk overestimated. Radiology 240:613-614

83. Hurwitz LM, Yoshizumi TT, Goodman PC et al (2009) Radiation dose savings for adult pulmonary embolus 64-MDCT using bismuth breast shields, lower peak kilovoltage, and automatic tube current modulation. AJR Am J Roentgenol 192:244-253

84. Vollmar SV, Kalender WA (2008) Reduction of dose to the female breast in thoracic CT: a comparison of standard-protocol, bismuthshielded, partial and tube-current-modulated CT examinations. Eur Radiol 18:1674-1682

85. Brisse HJ, Robilliard M, Savignoni A et al (2009) Assessment of organ absorbed doses and estimation of effective doses from pediatric anthropomorphic phantom measurements for multidetector row CT with and without automatic exposure control. Health Phys 97:303-314

86. Webb JA, Thomsen HS, Morcos SK (2005) The use of iodinated and gadolinium contrast media during pregnancy and lactation. Eur Radiol 15:1234-1240

87. Schaefer-Prokop C, Prokop M (2008) CTPA for the diagnosis of acute pulmonary embolism during pregnancy. Eur Radiol 18:2705-2708

88. Schueller-Weidekamm C, Schaefer-Prokop CM, Weber M, Herold CJ, Prokop M (2006) CT angiography of pulmonary arteries to detect pulmonary embolism: improvement of vascular enhancement with low kilovoltage settings. Radiology 241:899-907

89. Szucs-Farkas Z, Kurmann L, Strautz T, Patak MA, Vock P, Schindera ST (2008) Patient exposure and image quality of lowdose pulmonary computed tomography angiography: comparison of 100 - and $80-\mathrm{kVp}$ protocols. Invest Radiol 43:871-876

90. Matsuoka S, Hunsaker AR, Gill RR et al (2009) Vascular enhancement and image quality of MDCT pulmonary angiography in 400 cases: comparison of standard and low kilovoltage settings. AJR Am J Roentgenol 192:1651-1656

91. Forbes KP, Reid JH, Murchison JT (2001) Do preliminary chest $\mathrm{X}$-ray findings define the optimum role of pulmonary scintigraphy in suspected pulmonary embolism? Clin Radiol 56:397-400

92. Gleeson FV, Turner S, Scarsbrook AF (2006) Improving the diagnostic performance of lung scintigraphy in suspected pulmonary embolic disease. Clin Radiol 61:1010-1015

93. Harris B, Bailey DL, Roach PJ et al (2008) A clinical comparison between traditional planar V/Q images and planar images generated from SPECT V/Q scintigraphy. Nucl Med Commun 29:323-330

94. Bajc M, Olsson CG, Olsson B, Palmer J, Jonson B (2004) Diagnostic evaluation of planar and tomographic ventilation/ perfusion lung images in patients with suspected pulmonary emboli. Clin Physiol Funct Imaging 24:249-256

95. Collart JP, Roelants V, Vanpee D et al (2002) Is a lung perfusion scan obtained by using single photon emission computed tomography able to improve the radionuclide diagnosis of pulmonary embolism? Nucl Med Commun 23:1107-1113

96. Reinartz P, Wildberger JE, Schaefer W, Nowak B, Mahnken AH, Buell U (2004) Tomographic imaging in the diagnosis of pulmonary embolism: a comparison between V/Q lung scintigraphy in SPECT technique and multislice spiral CT. J Nucl Med 45:1501-1508 